\title{
Simulation of perspiration in sweating fabric manikin-Walter
}

\author{
J. Fan \\ Institute of Textiles and Clothing, \\ The Hong Kong Polytechnic University, Hong Kong
}

\begin{abstract}
Thermal manikins are essential tools for the comfort evaluation of the thermal environment and clothing as well as research into the dynamics of heat and moisture transfer from the human body to the environment - a foundation for thermal physiology and environmental engineering.

Since the development of the first non-sweating, single segment copper thermal manikin by the US Army in 1940s, there have been numerous developments worldwide in thermal manikin technologies. Nevertheless, the simulation of perspiration has continued to be one of the major challenges. Inspired by the thermoregulation system of the human body, a novel sweating fabric manikin nicknamed "Walter" has been developed in Hong Kong Polytechnic University. The new sweating manikin achieved perspiration over the entire body and a very high measurement accuracy (viz. a CV of less 5\%), yet with a cost of only a small fraction of other sweating manikins developed so far.
\end{abstract}

Keywords: sweating, perspiration, manikin, thermal comfort, clothing, environmental ergonomics.

\section{Introduction}

Comfortable clothes can be a matter of life or death in hazardous environments from wind-blasted mountain peaks and frozen Arctic wastes to space exploration. But thermal comfort also affects our everyday lives. Adequate insulation and good breathability are keys to comfort. In order to develop and optimize the functional design of clothing, it is essential to develop advanced 
tools for the evaluation of clothing thermal comfort, and for the investigation of the dynamic thermal interaction between human body, clothing and environment.

Thermal comfort of clothing systems may be evaluated by subjective wearer trials or objective simulation tests. Subjective wearer trials can relate the results directly to the clothing in actual use, but tend to be inconsistent and costly and can sometimes expose the subjects to danger when testing under extreme conditions. Objective simulation tests include flat plate methods (e.g. Togmeter, KESF Thermolab), cylindrical methods (e.g. Cylindrical Togmeter) and thermal manikins. Flat plate and cylindrical methods are useful for evaluating the thermal properties of clothing materials and simple clothing assemblies, but have difficulties in applying the results to the actual clothing systems in use. Thermal manikins have therefore been considered as the most useful tools for evaluating the thermal comfort of clothing systems [1].

The development of thermal manikins started during the Second World War. It is estimated that there are over 100 thermal manikins in the world [2]. Some of the most advanced recent manikins are divided into many sections and the temperature of each section is controlled independently so as to achieve a distribution similar to that of the human skin. Sweating is simulated by supplying water through the tiny tubes to the holes distributed at the manikin surface. Such manikins are very costly due to the complicated control systems for heating and water supply. The development cost for the sweating manikin- "SAM" in Switzerland is said to be 10 million Swiss Franc [3]. Nevertheless, the simulation of sweating in the existing manikins is limited by the practically manageable number of water supplying tubes and holes, which are far less than the approximately six million sweating glands on human. Besides, they are not very accurate because of the difficulty in maintaining consistent sweating and in accurately determining the humidity at the skin surface [4]. They also have very different thermal properties from that of a human body.

The new invention-Walter is inspired by the thermoregulation system of human body. With the original use of a breathable fabric to simulate the skin and a water circulation system to simulate the blood circulation, the novel manikin achieved perspiration over the entire body and very high measurement accuracy (viz. a CV of less $5 \%$ ), yet with a cost of only a small fraction of other sweating manikins developed so far.

\section{Description of sweating manikin-Walter}

The invention of "Walter" is based on a completely new concept. "Walter" is the first thermal manikin made of mainly water and high strength breathable fabric. Sweating is simulated by a waterproof, but moisture permeable, fabric 'skin' that holds the water, but allow moisture transmission from the manikin's insides through the millions of tiny pores in the skin. Walter simulates human thermal physiology. The core of Walter's body is controlled at $37^{\circ} \mathrm{C}$ and the body temperature regulation is achieved by regulating the rate of the pumps which supply warm water from the core region to the extremities. Simultaneous heat loss and evaporative water loss from the manikin are accurately measured, hence 
it takes only one step to measure the two most important parameters - thermal insulation and moisture vapour resistance. The arms and legs of "Walter" can be motorized to simulate walking motion. The perspiration rate can be regulated by changing the skin temperature and having a fabric skin of different moisture permeability. Since it mainly consists of water, it has a similar weight and heat capacity to a human body, which is also composed of mainly water. Figure 1 shows the front view of "Walter". The main dimension of "Walter" is listed in Table 1.

Table 1: Dimensions of Walter.

\begin{tabular}{|l|l|}
\hline Height & $172 \mathrm{~cm}$ \\
\hline Neck Circumference & $45 \mathrm{~cm}$ \\
\hline Chest Circumference & $95 \mathrm{~cm}$ \\
\hline Waist Circumference & $89 \mathrm{~cm}$ \\
\hline Hip Circumference & $100 \mathrm{~cm}$ \\
\hline Surface Area & $1.79 \mathrm{~m}^{2}$ \\
\hline
\end{tabular}

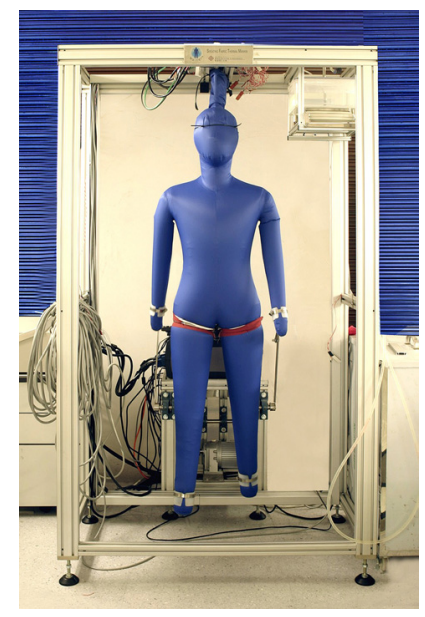

Figure 1: Front view of Walter.

"Walter" consists of the following sub-systems:

- Water circulation system.

- System for the simulation of "walking" motion

- Online water supply system.

- Control and measurement system

\subsection{Breathable fabric skin}

The "skin" of the manikin is very crucial to the success of this design. It should not only be able to produce the "perspiration", but also to hold as much as $70 \mathrm{~kg}$ of water inside the body and allow flexing induced by the body movement. According to our requirements, a special waterproof and moisture permeable fabric was used. The fabric has a sandwich structure. Its face side is a strong nylon fabric, inner side a protective knitting fabric and the middle a patented microporous polytetrafloroethene (PTFE) membrane. The membrane has billions of pores per square inch with the largest being less than $0.2 \mu \mathrm{m}$. The combination of the natural hydrophobicity of the PTFE polymer and the very small pore size and the great number of pores allows the membrane to discriminate between water as a gas and water as a liquid [5]. Since the water vapor molecular diameter is $2.72 \times 10-8 \mathrm{~cm}$, smaller than nitrogen gas molecular 
$(3.16 \times 10-8 \mathrm{~cm})$ and oxygen gas molecular $(2.96 \times 10-8 \mathrm{~cm})$, and is about 700 times smaller than a pore in the membrane. Liquid water on the other hand has tens of thousands of these molecules bonded together because of free energy considerations. In its smallest configuration, liquid water as a macro droplet is many thousands of times larger than the pores in the membrane. The membrane takes advantage of the difference of water in the state of gas and liquid to discriminate between the two so as to keep water in the manikin body and to allow moisture transmission through the "skin" to generate gaseous "perspiration".

\subsection{Water circulation system}

Just like human body's blood circulation system, "Walter" has a water circulation system which distributes the heat produced in the core region to the head, arms and legs. Temperatures at extremities depend on the water flow rates into them. The water flow rates can be adjusted by altering the five valves connecting to the head, arms and legs regions and the four small valves at the ends of the legs and arms. The five valves connecting to the head, arms and legs regions should be pre-adjusted, but the four small valves at the ends of the legs and arms can be adjusted during operation. The skin temperature can be further regulated in real time by a control software, which adjusts the rate of the pumps through regulating the AC power supply to the pumps.



Figure 2: The relationship between the mean skin temperature and AC frequency of the pumps.

\subsection{Simulation of "walking" motion}

"Walter" has a soft body, the arms can be bent easily when pushed or pulled in a simulating "walking" motion. The legs are however more difficult to bend due to their large cross-section. Joints between the legs and the torso should therefore be designed. The design idea of the joints is to reduce the cross-section at the connection between the legs and the torso so that the legs can be bent more easily, when pushed and pulled in simulating "walking" motion, but not blocking the water flow into and out of the legs. To achieve this idea, two plastic ABS 
rings are placed inside the body and one stainless steel ring is placed outside the skin on each leg. The inner plastic rings were used to keep the shape of the upper parts of the legs, whereas the external steel rings were used to limit the cross sectional area of the joints so as to facilitate bending. The diameter of the stainless steel ring is small than that of the ABS rings and it can be open and closed, so that the stainless steel ring could be inserted into the joint and used to reduce the cross-section of joint.

A quadric crank mechanism is used to create a forward and backward movement to push and pull the arms and legs to simulate "walk" motion. The "walking" pace can be adjusted by changing the position of the connection points of the cantilever. The pace size can be changed from 0.3 to $0.6 \mathrm{~m}$. The "walking" speed can be automatically changed by changing the frequency of the AC power supply to the driving motor using a computer controlled AC frequency regulator. The "walking" speed can vary from 0 to $3.6 \mathrm{~km} / \mathrm{hr}$.

\subsection{Online water supply system}

The fully filled water in the body of Walter maintains the body shape, skin temperature and the water evaporation from the skin of "Walter". Due to the large amount of water evaporated from "Walter" during its relatively long-time measurement cycle, an online automatic water supply system is necessary to compensate the water loss from "Walter", and to keep "Walter" at consistent working condition. This system is also critical to achieving online measurement of "Walter" system.

The system consists of a small water container and a programmable electronic balance. The water container was connected to "Walter's" body by a soft tube through siphon action. Figure 3 illustrates the online water supply system of "Walter".

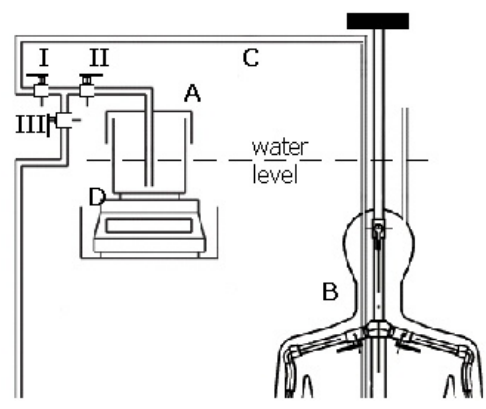

Figure 3: The automatic water supply system.

The water level in the water container is the same as that in the "Walter's" body at any time because of siphon action. Therefore the water in the water container can automatically flow into "Walter" to compensate the water loss 
from "Walter". The amount of water compensated to "Walter" can be used to calculate the evaporative rate of water from the skin of "Walter".

\subsection{Control and measurement system}

Figure 4 illustrates the control and measurement system of "Walter".

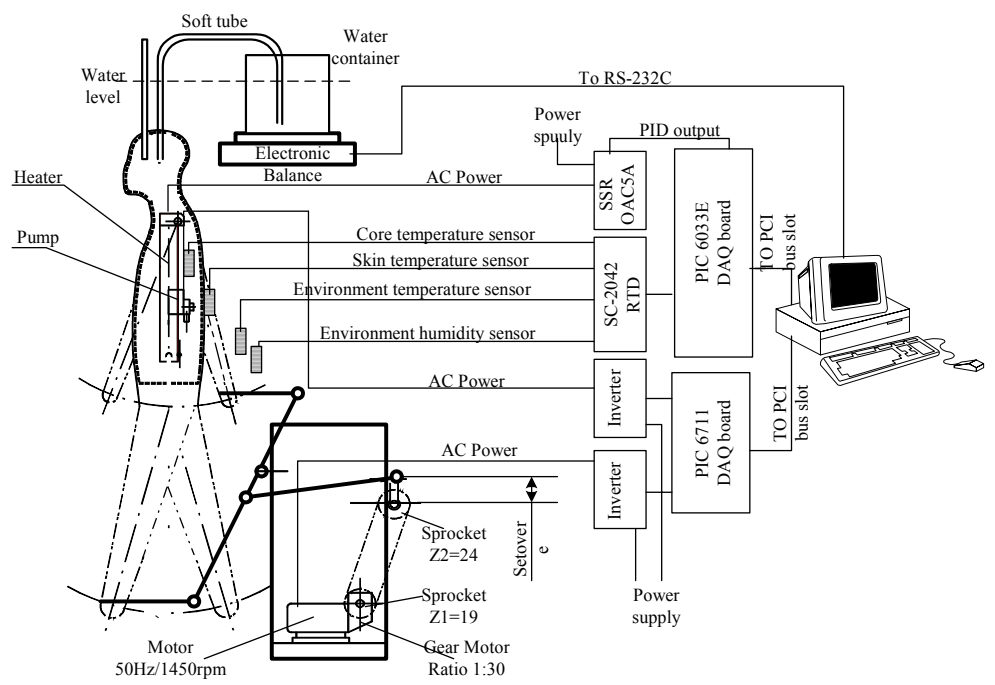

Figure 4: Control and measurement system of "Walter".

With this sub-system, the core temperature or the mean skin temperature of the manikin can be controlled automatically to the precision of $\pm 0.05^{\circ} \mathrm{C}$. The power supply (or heat loss), skin temperature at different positions and evaporative water loss can be measured in real time. Instead of weighing the entire manikin (about $70 \mathrm{~kg}$ ) for measuring the evaporative water loss which would be less accurate, "Walter" measures the water loss from a container (less than $6 \mathrm{~kg}$ ), which is connected to the manikin through a soft tube and the water level in the manikin is the same as that in the container as a result of siphon action. This greatly increased the measurement accuracy [6].

\subsection{Measurement using Walter}

By measuring the heat supply, evaporative water loss, skin temperatures at different locations, the two most important thermal comfort properties of clothing, viz. the thermal insulation (Rt) and moisture vapour resistance (Ret), can be calculated using the following formulae:

$$
R_{t}=\frac{A_{s}\left(\bar{T}_{s}-T_{a}\right)}{H_{s}+H_{p}-H_{e}}
$$




$$
\begin{gathered}
H_{e}=\lambda \cdot Q \\
R_{e t}=\frac{A_{s}\left(P_{s}^{*}-R H_{a} P_{a}^{*}\right)}{H_{e}}-R_{e s}
\end{gathered}
$$

where, $A_{s}$ is the total surface area of the manikin, $\bar{T}_{s}$ is the mean skin temperature, $T_{a}$ is the mean temperature of the environment, $H_{s}$ is the heat supplied to the manikin or the heat generated by the heaters, $H_{p}$ is the heat generated by the pump $\left(H_{p}=12.6 \mathrm{~W}\right), H_{e}$ is the evaporative heat loss from the water evaporation, $\lambda$ is the heat of evaporation of water at the skin temperature. From the data-book of thermal physics, $\lambda=0.67 \mathrm{~W} . \mathrm{h} / \mathrm{g}$ at $34^{\circ} \mathrm{C} . \quad \mathrm{Q}$ is the "perspiration" rate or water loss per unit time, which is measured automatically through the electronic balance, $P_{s}^{*}$ is the saturated water vapour pressure at the skin temperature, $R H_{a}$ the relative humidity of the surrounding environment, $P_{a}^{*}$ the saturated water vapour pressure at the surrounding environment, and $R_{e s}$ is the moisture vapour resistance of the "skin". $R_{e s}$ is predetermined constant for each type of fabric skin. For a normal skin used in our experiment, $R_{e s}$ equals to $8.6 \mathrm{~m} 2 \mathrm{~Pa} / \mathrm{W}[6]$.

From the thermal insulation (Rt) in $\mathrm{m}^{2 \circ} \mathrm{C} / \mathrm{W}$ and moisture vapour resistance (Ret) in $\mathrm{m}^{2} \mathrm{~Pa} / \mathrm{W}$, one further derive the moisture permeability index Im by the following formula (Based on ISO9920) :

$$
I_{m}=60.6 \times \frac{R_{t}}{R_{e t}}
$$

The moisture permeability index $\mathrm{Im}$ is a dimensionless measure of the moisture permeability of the clothing system.

By weighing the garments before putting on "Walter" and after taking off from Walter after a specified period, one can also determine the percentage of moisture accumulation within clothing by the following formula:

$$
M_{a}=\frac{W_{a}-W_{b}}{W_{b}} \times 100 \%
$$

The thermal insulation (Rt), vapour resistance (Ret), moisture permeability index (Im) and moisture accumulation (Ma) are important measures of clothing thermal comfort and can be interpreted in general as follows:

- The thermal insulation (Rt) should be as small as possible for summer clothing to keep cool;

- The thermal insulation (Rt) should be as high as possible for winter clothing to keep warm;

- The vapour resistance (Ret) of clothing should be as low as possible for any type of clothing to make the clothing permeable;

- The moisture permeability index (Im )should be as high as possible for any type of clothing to make the clothing permeable;

- The moisture accumulation Ma should be as low as possible for any type of clothing to keep the skin and clothing dry. 


\section{Results and discussion}

\subsection{The accuracy and reproducibility of Walter}

The thermal insulation and moisture vapor resistance of the nude manikin were tested repeatedly for three times in different wind velocity under the $20^{\circ} \mathrm{C}$ and $50 \%$ RH. The mean values are listed in Table 2 and the standard deviation of each value is listed in Table 3.

Table 2: $\quad$ The mean values of Ioa and Roa vary with wind velocity.

\begin{tabular}{|l|l|l|l|l|l|l|l|}
\hline $\begin{array}{l}\text { Wind } \\
\text { velocity } \\
(\mathrm{m} / \mathrm{s})\end{array}$ & Ts & Te & $\mathrm{He}$ & $\mathrm{W}$ & $\mathrm{Q}$ & Ioa & Roa \\
\hline 0.22 & 35.04 & 20.32 & 46.76 & 649.90 & 562.59 & 0.101 & 12.90 \\
\hline 0.85 & 34.91 & 19.75 & 48.25 & 842.95 & 697.00 & 0.076 & 8.62 \\
\hline 1.69 & 34.96 & 19.83 & 47.68 & 1046.46 & 833.76 & 0.058 & 5.86 \\
\hline 2.48 & 34.82 & 19.89 & 53.87 & 1157.64 & 874.55 & 0.049 & 4.61 \\
\hline 3.12 & 34.89 & 19.79 & 52.24 & 1271.96 & 916.97 & 0.043 & 4.19 \\
\hline 4.04 & 34.94 & 19.84 & 49.33 & 1415.32 & 1004.19 & 0.038 & 3.29 \\
\hline
\end{tabular}

Table 3: $\quad$ The standard deviation of Ioa and Roa measured on the manikin.

\begin{tabular}{|l|l|l|l|l|l|l|l|}
\hline $\begin{array}{l}\text { Wind } \\
\text { velocity } \\
(\mathrm{m} / \mathrm{s})\end{array}$ & Ts & Te & He & W & Q & Ioa & Roa \\
\hline 0.22 & 0.02 & 0.01 & 0.50 & 4.71 & 6.63 & 0.003 & 0.32 \\
\hline 0.85 & 0.01 & 0.02 & 0.10 & 1.88 & 5.43 & 0.001 & 0.13 \\
\hline 1.69 & 0.01 & 0.17 & 0.35 & 0.01 & 6.18 & 0.001 & 0.11 \\
\hline 2.48 & 0.05 & 0.03 & 0.02 & 18.28 & 3.48 & 0.001 & 0.01 \\
\hline 3.12 & 0.02 & 0.01 & 0.41 & 10.32 & 5.67 & 0.000 & 0.01 \\
\hline 4.04 & 0.01 & 0.01 & 0.51 & 1.56 & 6.87 & 0.000 & 0.01 \\
\hline
\end{tabular}

The average thermal insulation of the nude manikin in the case of standing in still air was found to be $0.101 \mathrm{~m}^{20} \mathrm{C} / \mathrm{W}$ with a standard deviation of 0.003 (viz. $\mathrm{CV}=3 \%$ ), the average moisture vapor resistance of nude manikin is $12.90 \mathrm{~m}^{2} \mathrm{~Pa} / \mathrm{W}$ with a standard deviation of 0.32 (viz. $\mathrm{CV}=2.5 \%$ ). These results show that the accuracy of "Walter" is very high.

A clothing ensembles include underwear and outwear are used to valid the reproducibility. The experiment conducts three independent replications of thermal measurements. The independent replication means taking the all garments off and putting them back on for each replication of the test. The test 
results are listed in Table 4. The $\mathrm{CV}$ of the repeated measurements of the clothing thermal insulation was $3.3 \%$ and that of the moisture vapour resistance is $2.2 \%$, which are very good.

Table 4: $\quad$ The reproducibility of measurements on manikin.

\begin{tabular}{|l|l|l|l|l|l|l|l|}
\hline & $\mathrm{Ts}$ & $\mathrm{Te}$ & $\mathrm{He}$ & $\mathrm{W}$ & $\mathrm{Q}$ & $\mathrm{Rt}$ & $\mathrm{Re}$ \\
\hline Repeat1 & 35.14 & 20.31 & 49.22 & 314.5 & 279.7 & 0.218 & 34.06 \\
\hline Repeat2 & 35.01 & 20.38 & 49.25 & 318.5 & 277.9 & 0.206 & 33.89 \\
\hline Repeat3 & 35.13 & 20.31 & 49.43 & 315.3 & 271.2 & 0.206 & 35.32 \\
\hline mean & 35.09 & 20.33 & 49.30 & 316.12 & 276.27 & 0.210 & 34.42 \\
\hline Stdev & 0.07 & 0.04 & 0.12 & 2.14 & 4.50 & 0.007 & 0.78 \\
\hline
\end{tabular}

\subsection{Comparison with previously reported results}

The results are generally comparable to those reported elsewhere. For example, the thermal insulation of the nude manikin standing in the almost still air was found to be $0.101 \mathrm{~m}^{20} \mathrm{C} / \mathrm{W}$, which is within the range of the variations (from 0.051 to $0.104 \mathrm{~m}^{20} \mathrm{C} / \mathrm{W}$ ) found in an inter-laboratory study [7]. The moisture vapour resistance of the nude manikin obtained in our experiments is $12.9 \mathrm{~m}^{2} \mathrm{~Pa} / \mathrm{W}$, which is at the lower end of the reported range of from 11.0 to $20.0 \mathrm{~m}^{2} \mathrm{~Pa} / \mathrm{W}$ [7]. The actual relative humidity at the skin surface of those sweating manikins reported in the previous the inter-laboratory study might be lower than the assumed value of $100 \%$, which might make the values of the moisture vapour resistances greater than the actual ones.

\section{Conclusions}

Through the simulation of the human skin using a breathable fabric, the simulation of the human thermal regulation system using a water circulation and temperature control system and the simulation of human body using water, the new invention has demonstrated distinct advantages in terms of its physical characteristics and measurement accuracy. The invention is a breakthrough in manikin technology and has found important applications in comfort evaluation of functional clothing and research in thermal physiology and environmental engineering.

\section{References}

[1] Fan, J. \& Chen, Y. S. Measurement of Clothing Thermal Insulation and Moisture Vapour Permeability Using a Novel Perspiring Fabric Thermal Manikin, Measurement Science and Technology, 13, pp. 1115-1123, 2002. 
230 Design and Nature III: Comparing Design in Nature with Science and Engineering

[2] Holmer, I. Thermal manikin history and application, European Journal of Applied Physiology, 92(6), pp. 614-618, 2004.

[3] Richard, M. Private communication during the 4th international meeting on thermal manikins, September 2001, St. Gallen, Switzerland.

[4] Richard M., Mattle N. \& Becker C. Assessment of the protection and comfort of fire fighter's clothing using a sweating manikin, Proceedings of the 5th European Conference on Protective Clothing and NOKOBETEF7, May 2003, Montreux, Switzerland.

[5] Tanner, J. C. Breathability, Comfort and GORE-TEX Laminates, Journal of Coated Fabrics, 8, pp. 312, 1979.

[6] Fan, J. \& Qian, X. New Functions and Applications of Walter, the Sweating Fabric Manikin, European Journal of Applied Physiology, 92, pp. 641-644, 2004.

[7] McCullough, E. A., Interlaboratory Study of Sweating Manikins, Proceedings of the Fourth International Meeting on Thermal Manikins, EMPA, Switzerland, 27-28, September 2001. 